\title{
Factors influencing pregnancy outcome in women with vaginal bleeding before midpregnancy: a prospective case control study
}

\author{
Shyamala Guruvare*, Priyanka Medipalli, Srinivasa M. Urala, \\ Lavanya Rai, Shripad Hebbar, Prashanth Adiga
}

Department of Obstetrics \& Gynecology, Kasturba Medical College, Manipal-576104, Karnataka, India

Received: 01 March 2015

Accepted: 19 April 2015

\section{*Correspondence:}

Dr. Shyamala Guruvare,

E-mail: shyamala_doc@yahoo.co.in, shyamalarajgopal@hotmail.com

Copyright: () the author(s), publisher and licensee Medip Academy. This is an open-access article distributed under the terms of the Creative Commons Attribution Non-Commercial License, which permits unrestricted non-commercial use, distribution, and reproduction in any medium, provided the original work is properly cited.

\begin{abstract}
Background: Pregnancy complications are observed to be higher among women with first trimester bleeding compared to those without bleeding. We intended to compare the obstetric outcome based on details of bleeding episodes, i) in groups with spotting, bleeding and brown discharge; ii) in those with or without subchorionic haemorrhage; and iii) between single versus recurrent episodes of bleeding.

Methods: We conducted a prospective case control study involving the women enrolled for antenatal care before 20 weeks of gestation. Information was collected regarding the details of bleeding, the pregnancy complications and the perinatal outcome. The outcome variables were analysed as per the study objectives using SPSS version 16.0.

Results: Pregnancies complicated by bleeding before 20 weeks of gestation were associated with the significant increase in preterm premature rupture of the membranes [P 0.045; RR 4.8 (95\% CI 1.5-15)], intrauterine growth restriction [P 0.039; RR 2.5(95\% CI 1.1-6.3)], oligohydramnios [RR 4.3 (95\% CI 2-9)], hypertension [RR 1.8 (95\% CI 1.07-3.09)], small for gestational age babies [P 0.047; RR 2.7 (95\% CI 1.2-6.1)] and NICU admissions [P 0.016; RR 2.7 (95\% CI 1.1-6.5)]. Fresh bleeding/ spotting were associated with increased pregnancy complications (P 0.048) compared to brownish discharge. Presence of subchorionic hemorrhage on ultrasound in women with vaginal bleeding was associated with increased pregnancy complications (P 0.044). No difference in terms of complications, mean gestational age and mean birth weight was noted depending on the number of episodes of bleeding.

Conclusions: Fresh bleeding and presence of subchorionic hemorrhage predict a poor pregnancy outcome in women with bleeding before 20 weeks of pregnancy.
\end{abstract}

Keywords: Vaginal bleeding, Pregnancy complications, Obstetric outcome, Subchorionic hemorrhage

\section{INTRODUCTION}

Bleeding in early trimester is associated with several pregnancy complications and poor perinatal outcome. Several studies have found association between preterm labor, preeclampsia, fetal growth restriction, antepartum hemorrhage and related poor perinatal outcome. ${ }^{1,2}$

With this background, we conducted this study with an extended intension of finding the factors in early trimester bleeding which adversely influence pregnancy outcome.

\section{Objectives of the study}

We intended to compare the maternal and perinatal outcome based on details of bleeding episodes, i) in groups with spotting, bleeding and brown discharge; ii) in those with or without subchorionic haemorrhage; and iii) between single versus recurrent episodes of bleeding. 


\section{METHODS}

We conducted a prospective nested case control study study involving the women enrolled for antenatal care before 20 weeks of gestation after ultrasound confirmation of fetal cardiac activity. Institutional ethical committee approval was obtained.

As the fetal growth restriction was the most predominant poor outcome expected, we considered prevalence of small for gestational age (SGA) for calculation of sample size. Narang et al determined that $3.53 \%$ of the neonates were small for gestational age (SGA). ${ }^{3}$

We assumed that any obstetric event significantly influences the occurrence of SGA if it can alter the above incidence by $5 \%$. Based on this information, first we calculated the required number of cases to obtain the significance level of 0.05 using the formula:

$$
n=\frac{Z^{2} p(1-p)}{d^{2}}
$$

Wherein; $Z=1.96$ (the approximate value of the 97.5 percentile point of the normal distribution for significance level of 0.05 or $5 \%), p$ is the prevalence of SGA $(3.53 \%$ or 0.0353$), d$ is the precision level $(5 \%$ or 0.05 , also known as acceptable margin of error). Accordingly the minimum sample size to prove null hypothesis was calculated to be 53. Our sample size of cases was 67 which were far satisfactory.

To improve the power of study further we kept the case to control ratio 1:4 (total number of cases in controls 268) as recommended by Biesheuvel et al. ${ }^{4}$

Women with bleeding due to local causes like - fibroid polyp, carcinoma cervix, cervical erosion and those who had spontaneous abortions before 20 weeks were excluded from the study.

In women with vaginal bleeding details regarding the nature of bleeding- fresh or brownish bleeding, quantity of bleeding, number of episodes of bleeding was noted. At antenatal visits serial growth scans were done; the complications were managed as per the standard protocol. Obstetric outcome measures: preterm labor, Preterm Premature Rupture of Membranes (PPROM), fetal growth restriction (IUGR), oligohydramnios, hypertension in pregnancy, antepartum hemorrhage (APH) and placental weight; perinatal outcome measures such as birth weight of baby, APGAR score at birth, NICU admission, neonatal deaths were noted.

Women complaining of vaginal bleeding before 20 weeks of gestation were (cases) compared against women without vaginal bleeding (controls). In women with vaginal bleeding, we looked for correlation of the poor outcome with the number of episodes of bleeding before 20 weeks, nature of bleeding (fresh bleeding/brownish discharge) and presence or absence of subchorionic haemorrhage on ultrasound.

Statistical analysis was done using SPSS 16.0; Pearson Chi Square test was used to calculate the statistical significance and relative risks.

\section{RESULTS}

Out of 76 women with vaginal bleeding before 20 weeks of pregnancy, nine had spontaneous abortions and were excluded. There was none with local cause for bleeding. Maternal and perinatal outcome in the remaining 67 women was compared with 268 women without early trimester bleeding. The mean age and parity index in both groups were comparable. There was no significant difference in the presence of pre-existing illness in both the groups (Table 1).

Table 1: Maternal profile in in women with vaginal bleeding before 20 weeks of gestation to those without bleeding.

\begin{tabular}{|llll|}
\hline & $\begin{array}{l}\text { Bleeding } \\
\text { before 20 } \\
\text { weeks }(\mathrm{n}=67) \\
\mathrm{n}(\%)\end{array}$ & $\begin{array}{l}\text { No bleeding } \\
(\mathrm{n}=268)\end{array}$ & P value \\
$\mathrm{n}(\%)$ & & \\
\hline $\begin{array}{l}\text { Mean maternal } \\
\text { age (years) }\end{array}$ & 27.9 & 27.5 & 0.736 \\
\hline $\begin{array}{c}\text { Parity index } \\
\text { Primi } \\
\text { Multi }\end{array}$ & $44(65.7)$ & $155(57.8)$ & 0.243 \\
\hline $\begin{array}{l}\text { Pre-existing } \\
\text { illness }\end{array}$ & $1(1.5)$ & $15(5.6)$ & 0.584 \\
\hline
\end{tabular}

Fetal growth restriction, oligohydramnios, PPROM, hypertension were more in women who had early trimester bleeding compared to those without such history (Table 2). There was no significant increase in prevalence of preterm labour and antepartum haemorrhage in women with bleeding compared to women without bleeding before 20 weeks of gestation. However preterm labour before 32 weeks of gestation were higher [ 3 of $6(50 \%)$ ] in women with bleeding compared to those without bleeding [2 of $15(13 \%)]$. Out of 3 cases with APH two had placental abruption, one had placenta previa.

Small for gestation babies were obviously more in the group with bleeding. Also more newborns in this group had poor APGAR scores at birth and required NICU care. Mean birth weight (2500 g versus 2819), placental weight ( $471 \mathrm{~g}$ versus $489 \mathrm{~g}$ ) and mean gestational age at delivery (36 weeks versus 37 weeks) did not differ between the groups. 
Table 2: Comparison of obstetric outcome in women with vaginal bleeding before 20 weeks of gestation to those without bleeding.

\begin{tabular}{|c|c|c|c|c|c|}
\hline Outcome variable & $\begin{array}{l}\text { Women with } \\
\text { vaginal bleed } \\
\text { (n=67) } \\
\mathbf{N}(\%)\end{array}$ & $\begin{array}{l}\text { Women without } \\
\text { vaginal bleed } \\
\text { (n=268) } \\
\mathbf{N}(\%)\end{array}$ & $\begin{array}{l}\text { Significance } \\
\text { P value }\end{array}$ & $\begin{array}{l}\text { Relative } \\
\text { risk }\end{array}$ & $\begin{array}{l}95 \% \\
\text { confidence } \\
\text { interval }\end{array}$ \\
\hline Preterm labor & $6(9)$ & $15(5.6)$ & 0.310 & 1.6 & $0.64-3.9$ \\
\hline PPROM & $6(9)$ & $5(1.9)$ & 0.004 & 5.1 & $1.5-15$ \\
\hline Fetal growth restriction & $7(10.4)$ & $11(4.1)$ & 0.039 & 2.5 & $1.1-6.3$ \\
\hline Antepartum hemorrhage & $3(4.5)$ & $8(3)$ & 0.540 & 1.5 & $0.4-5.5$ \\
\hline Hypertension in pregnancy & $16(4.5)$ & $35(3)$ & 0.027 & 1.8 & $1.07-3.09$ \\
\hline Oligohydramnios & $13(19.4)$ & $12(4.5)$ & $<0.001$ & 4.3 & $2-9$ \\
\hline SGA & $10(16.7)$ & $16(7.1)$ & 0.047 & 2.7 & $1.2-6.1$ \\
\hline Poor APGAR & $62(83.3)$ & $248(92.9)$ & 0.058 & 4.1 & $0.8-1.9$ \\
\hline Stillbirth & $2(3)$ & $4(1.5)$ & 0.410 & 2 & $0.3-10$ \\
\hline NICU admission & $8(12.5)$ & $10(4.5)$ & 0.016 & 2.7 & $1.1-6.5$ \\
\hline
\end{tabular}

Chi Square test

Women with fresh bleeding/ spotting had higher incidence of pregnancy complications compared to those with brownish discharge. However there was no significant difference in mean birth weight and mean gestational age at delivery depending on the type of bleeding (Table 3). There was no significant difference in pregnancy complications, mean birth weight, and mean gestational age at delivery depending on single or recurrent episodes of bleeding (Table 4). In women with early trimester bleeding the presence of subchorionic hemorrhage on ultrasound was associated with increased risk of pregnancy complications. However there was no difference in mean birth weight and mean gestational age at delivery depending on presence or absence of subchorionic hemorrhage (Table 5).

Table 3: Comparison of pregnancy outcome, mean birth weight and mean gestational age at delivery according to type of bleeding in women with vaginal bleeding before 20 weeks of gestation.

\begin{tabular}{|lllll|}
\hline & $\begin{array}{l}\text { Spotting } \\
(\mathrm{n}=43) \\
\mathrm{n}(\%)\end{array}$ & $\begin{array}{l}\text { Fresh } \\
\text { bleed } \\
(\mathrm{n}=15) \\
\mathrm{n}(\%)\end{array}$ & $\begin{array}{l}\text { Brownish } \\
\text { discharge } \\
(\mathrm{n}=9)\end{array}$ & $\begin{array}{l}\mathrm{P} \\
\mathrm{n}(\%)\end{array}$ \\
value \\
\hline $\begin{array}{l}\text { Poor } \\
\text { pregnancy } \\
\text { outcome }\end{array}$ & $28(65.1)$ & $10(66.7)$ & $2(22.2)$ & 0.048 \\
\hline $\begin{array}{l}\text { Mean birth } \\
\text { weight } \\
\text { (Grams) }\end{array}$ & 2537 & 2412 & 2918 & 0.23 \\
\hline $\begin{array}{l}\text { Mean } \\
\text { gestational age } \\
\text { at delivery } \\
\text { (Weeks) }\end{array}$ & 36.6 & 35.8 & 38.7 & 0.418 \\
\hline
\end{tabular}

Table 4: Comparison of pregnancy outcome, mean birth weight and mean gestational age at delivery depending on episodes of bleeding in women with vaginal bleeding before 20 weeks of gestation.

\begin{tabular}{|ll|ll|} 
& $\begin{array}{l}\text { Single } \\
(\mathrm{n}=55) \\
\mathrm{n}(\%)\end{array}$ & $\begin{array}{l}\text { Recurrent } \\
(\mathrm{n}=12)\end{array}$ & $\begin{array}{l}\mathrm{P} \\
\mathrm{n}(\%)\end{array}$ \\
value \\
\hline Poor pregnancy outcome & $32(58.2)$ & $8(66.7)$ & 0.587 \\
\hline Mean birth weight (Grams) & 2601 & 2375 & 0.256 \\
\hline $\begin{array}{l}\text { Mean gestational age at } \\
\text { delivery (Weeks) }\end{array}$ & 36.9 & 35.5 & 0.314 \\
\hline
\end{tabular}

Chi Square test

Table 5: Comparison of pregnancy outcome, mean birth weight and mean gestational age at delivery depending on presence or absence of subchorionic hemorrhage on ultrasound in women with vaginal bleeding before 20 weeks of gestation.

\begin{tabular}{|llll|}
\hline & $\begin{array}{l}\text { Subchorionic } \\
\text { hemorrhage } \\
(\mathrm{n=16}) \\
\mathrm{n}(\%)\end{array}$ & $\begin{array}{l}\text { No subchorionic } \\
\text { hemorrhage } \\
(\mathrm{n}=51)\end{array}$ & $\begin{array}{l}\mathrm{P} \\
\mathrm{n}(\%)\end{array}$ \\
\hline $\begin{array}{l}\text { Poor pregnancy } \\
\text { outcome }\end{array}$ & $13(81.3)$ & $27(52.9)$ & 0.044 \\
\hline $\begin{array}{l}\text { Mean birth } \\
\text { weight (Grams) }\end{array}$ & 2235 & 2662 & 0.194 \\
\hline $\begin{array}{l}\text { Mean } \\
\text { gestational age } \\
\text { at delivery } \\
\text { (Weeks) }\end{array}$ & 36.3 & 36.8 & 0.557 \\
\hline & & & \\
\hline
\end{tabular}

Chi Square test 


\section{DISCUSSION}

Early trimester bleeding is found to result in defective placentation and subsequently poor pregnancy outcome. Later pregnancy complications like fetal growth restriction, preeclampsia, preterm labour and preterm premature rupture rupture of membranes have been shown to be associated with impaired placentation and failure of physiological invasion of spiral arterioles. The increased risk of placenta previa, placental abruption and APH of unknown origin are also attributed to the problems with placental development. $^{2}$

A large population based cohort study in China showed that first trimester vaginal bleeding is associated with an increased risk of low birth weight, preterm delivery and small for gestational age babies. ${ }^{5}$ Similarly other studies also showed two fold increased risk of preterm delivery ${ }^{6,7}$ and 6.24-fold increased risk of spontaneous preterm labor $^{8}$ in association with early pregnancy bleeding. Women who had bleeding might have vaginal infections because of alteration in vaginal $\mathrm{pH}$. Besides, placental insufficiency also may make them susceptible for preterm labor. Though our study showed no association between overall preterm labor and early trimester bleeding there was significantly more preterm labor before 32 weeks of gestation in women with history of bleeding (3 of 6 $(50 \%)$ versus 2 of $15(13 \%)$. Ours being a hospital based cohort, the protocol of looking for vaginal infections and vigorously treating them with antimicrobials might have reduced the overall preterm labor in our group. Those who found increased risk of preterm delivery also observed that recurrent bleeding had stronger association single episode of bleeding. ${ }^{8,9}$ Lykke et al. also extended the observation that first-trimester bleeding in the first pregnancy increased the risk of recurrence in the second pregnancy from $2.2 \%$ to $8.2 \%$ (OR 4.05 ; $95 \%$ CI 3.78 4.34), preterm delivery from $2.7 \%$ to $4.8 \%$ (OR 1.83 ; $95 \%$ CI 1.67-2.00), and placental abruption from $0.9 \%$ to $1.0 \%$ (OR 1.29; 95\% CI 1.07-1.56) in the second pregnancy. ${ }^{9}$

Our study showed increased risk of hypertension in pregnancy (P 0.027 RR-1.8, 95 ${ }^{\text {th }}$ CI 1.07-3.09) but not preeclampsia-eclampsia. Similar to the previous reports ${ }^{2,10}$ we too observed that bleeding before 20 weeks was associated with abruption more than 2 times when compared to controls; there was no difference in the incidence of placenta previa and APH of unknown origin. This was in contrast with the study by Ajith Wijesiriwardana ${ }^{11}$ et al. which showed increased risk of APH of unknown origin in women with early trimester bleeding (RR-1.83, $95^{\text {th }}$ CI 1.73-2.01). There was only one postpartum haemorrhage in the group with early trimester bleeding who also had preeclampsia and fetal growth restriction.

In our study bleeding in early trimester was associated with significant risk of delivering small for gestational age babies [RR-2.7 $\left(95^{\text {th }} \mathrm{CI}-1.2-6.1\right)$ ]. Bleeding before 20 weeks was associated with 2.5 times increased risk of fetal growth restriction comparable with results in other studies; ${ }^{2,12}$ also there was more oligohydramnios with relative risk of 4.3. Though there was observable difference in mean birth weight and mean gestational age at delivery in both the groups (vaginal bleeding before 20 weeks of gestation was associated with 200 grams decrease in mean birth weight and 8 days difference in mean gestational age), it was not statistically significant. Unlike other studies, our group with bleeding did not have significantly increased prevalence of preeclampsiaeclampsia, preterm labor and placental abruption; this might be the reason for the above observation in mean birth weight and mean gestation age. Although our study showed more frequent still births in women with bleeding before 20 weeks of gestation, it was not statistically significant.

When the number of episodes of bleeding was considered, we did not find significant difference in obstetric complications, mean birth weight and mean gestational age at delivery in women with single or recurrent episodes of early trimester bleeding. This was in contrast with study by Yang et al. which showed bleeding of multiple episodes and more blood loss is associated with two fold increased risk of early preterm, PPROM and preterm labor. ${ }^{5}$

Presence of subchorionic hemorrhage on ultrasound in women with bleeding before 20 weeks of gestation was associated with more obstetric complications ( $\mathrm{P}$ value $0.048)$. This was comparable with other studies. ${ }^{13,14}$

Probably the presence of subchorionic hemorrhage indicates that the bleed is significant and placental insufficiency is probably more in them because of significant placental separation.

The merits of the study are that it was a prospective cohort study with a good ratio of case to controls taken based on the prevalence of the problem. The ratio of the cases and the controls of 1:4 was chosen after taking into account the prevalence of each. Each outcome variable is clearly defined. This has minimized the erroneous results and has increased the reliability of the results. We have also tried to find out the relative risks for the outcome variables. Besides comparing the cases and the controls, an effort was made to analyse the outcome among cases based on type of bleeding, number of episodes, presence/absence of subchorionic bleed on ultrasound.

However the limitations of the study are: 1. the individual outcome variables could not be evaluated between the subgroups of the cases because the number was not sufficient for that purpose; 2 . women with early trimester bleeding obviously received more intensive fetal surveillance compared to the controls. Some of the possible pregnancy complications might have been averted, thus resulting in better outcome (Example: Preterm labor, still birth rate). 


\section{CONCLUSION}

Pregnancies complicated by bleeding before 20 weeks of gestation are at increased risk of complications like PPROM, fetal growth restriction, oligohydromnios, hypertension and low birth weight newborns. Fresh bleeding/spotting is associated with increased risk of late pregnancy complications when compared to brownish discharge per vagina. Presence of subchorionic hemorrhage on ultrasound in women with bleeding before 20 weeks of gestation is associated with higher risk of complications.

\section{ACKNOWLEDGEMENTS}

We acknowledge the support of the faculty in the department of Obstetrics and Gynecology, Kasturba Medical College, Manipal for their guidance during the study

Funding: No funding sources Conflict of interest: None declared

Ethical approval: The study was approved by the institutional ethics committee

\section{REFERENCES}

1. Funderburk SJ, Guthrie D, Meldrum D. Outcome of pregnancies complicated by early vaginal bleeding. BJOG. 2005;87(2):100-5.

2. Saraswat L, Bhattacharya S, Maheshwari A, Bhattacharya S. Maternal and perinatal outcome in women with threatened miscarriage in first trimester. BJOG. 2010;117(3):245-57.

3. Narang A, Chaudhuri MK, Kumar P. Small for gestational age babies: Indian scene. Indian J Pediatr. 1997;64(2):221-4.

4. Biesheuvel CJ, Vergouwe Y, Oudega R, Hoes AW, Grobbee DE, Moons KG. Advantages of the nested case-control design in diagnostic research. BMC Med Res Methodol. 2008;8:48.

5. Sun L, Tao F, Hao J, Su P, Liu F, Xu R. First trimester vaginal bleeding and adverse pregnancy outcomes among Chinese women: from a large cohort study in China. J Matern Fetal Neonatal Med. 2012;25(8):1297-301.

6. Yang J, Hartmann KE, Savitz DA, Herring AH, Dole $\mathrm{N}$, Olshan AF, et al. Vaginal bleeding during pregnancy and preterm birth. Am J Epidemol. 2004;160:118-25.

7. Hossain R, Harris T, Lohsoonthorn V, Williams MA. Risk of preterm delivery in relation to vaginal bleeding in early pregnancy. Eur J Obstet Gynecol Reprod Biol. 2007;135:158-63.

8. Sharami SH, Faraji Darkhaneh R, Zahiri Z, Milani F, Asgharnia M, Shakiba M, et al. The relationship between vaginal bleeding in the first and second trimester of pregnancy and preterm labor. Iran $\mathrm{J}$ Reprod Med. 2013;11(5):385-90.

9. Lykke JA, Dideriksen KL, Lidegaard O, LanghoffRoos J. First-trimester vaginal bleeding and complications later in pregnancy. Obstet Gynecol. 2010;115(5):935-44.

10. Batzofin JH, Fielding WL, Friedman EA. Effect of vaginal bleeding in early pregnancy on outcome. Obstet Gynecol. 1984;63:515-8.

11. Wijesiriwardana A, Bhattacharya S, Shetty A, Smith $\mathrm{N}$, Bhattacharya S. Obstetric outcome in women with threatened miscarriage in first trimester. Obstet Gynecol. 2006;107:557-62.

12. Berkowitz GS, Harlap S, Beck GJ, Freeman DH, Balas M. Early gestational bleeding and pregnancy outcome: a multivariant analysis. Int J Epidemiol. 1983;12:165-73.

13. Maso G, D’Ottavio G, Seta F, Sartore A, Piccoli M, Mandruzzato G. First trimester intrauterine hematoma and outcome of pregnancy. Obstet Gynecol. 2005;105(2):339-44.

14. Nagy S, Bush M, Stone J, Lapinski RH, Gardo S. Clinical significance of subchorionic and retroplacental hematoma detected in first trimester of pregnancy. Obstet Gynecol. 2003;102(1):94-100.

DOI: $10.18203 / 2320-1770 . i j r \operatorname{cog} 20150059$

Cite this article as: Guruvare S, Medipalli P, Urala SM,

Rai L, Hebbar S, Adiga P. Factors influencing pregnancy outcome in women with vaginal bleeding before midpregnancy: a prospective case control study. Int $\mathbf{J}$ Reprod Contracept Obstet Gynecol 2015;4:601-5. 